\title{
Compressed Cliques Graphs, Clique Coverings and Positive Zero Forcing
}

\author{
Shaun Fallat ${ }^{\dagger} \quad$ Karen Meagher ${ }^{\ddagger} \quad$ Abolghasem Soltani ${ }^{\S}$ \\ Boting Yang
}

September 22, 2018

\begin{abstract}
Zero forcing parameters, associated with graphs, have been studied for over a decade, and have gained popularity as the number of related applications grows. In particular, it is well-known that such parameters are related to certain vertex coverings. Continuing along these lines, we investigate positive zero forcing within the context of certain clique coverings. A key object considered here is the compressed cliques graph. We study a number of properties associated with the compressed cliques graph, including: uniqueness, forbidden subgraphs, connections to Johnson graphs, and positive zero forcing.
\end{abstract}

Keywords: Positive zero forcing number, cliques, clique cover number, compressed cliques graph, Johnson graph, forbidden subgraphs.

AMS Subject Classifications: 05C50, 05C75, 05C69

\section{Introduction}

Suppose that $G$ is a simple finite graph with vertex set $V=V(G)$ and edge set $E=E(G)$. We use $\{u, v\}$ to denote an edge with endpoints $u$ and $v$. Further, for a graph $G=(V, E)$

${ }^{*}$ Department of Mathematics and Statistics, University of Regina, Regina, Saskatchewan S4S 0A2, Canada. Research supported in part by an NSERC Discovery Research Grant, Application No.: RGPIN2014-06036. Email: Shaun.Fallat@uregina.ca.

${ }^{\dagger}$ Corresponding Author

${ }_{\ddagger}^{\ddagger}$ Department of Mathematics and Statistics, University of Regina, Regina, Saskatchewan S4S 0A2, Canada. Research supported in part by an NSERC Discovery Research Grant, Application No.: RGPIN341214-2013. Email: Karen. Meagher@uregina.ca.

${ }^{\S}$ Department of Mathematics and Statistics, University of Regina, Regina, Saskatchewan S4S 0A2, Canada. Research supported in part by an NSERC Discovery Research Grant, Application No.: RGPIN2014-06036. Email: abel.soltani@gmail.com.

"Department of Computer Science, University of Regina, Regina, Saskatchewan S4S 0A2, Canada. Research supported in part by an NSERC Discovery Research Grant, Application No.: RGPIN-2013-261290. Email: Boting. Yang@uregina.ca. 
and $v \in V$, the vertex set $\{u:\{u, v\} \in E\}$ is the neighbourhood of $v$, denoted as $N_{G}(v)$, and the size of the neighbourhood of $v$ is called the degree of $v$. For $V^{\prime} \subseteq V$, the vertex set $\left\{x:\{x, y\} \in E, x \in V \backslash V^{\prime}\right.$ and $\left.y \in V^{\prime}\right\}$ is the neighbourhood of $V^{\prime}$, denoted as $N_{G}\left(V^{\prime}\right)$. Also, we let the set $N_{G}[v]=\{v\} \cup N_{G}(v)$ denote the closed neighbourhood of the vertex $v$. We use $G\left[V^{\prime}\right]$ to denote the subgraph induced by $V^{\prime}$, which consists of all vertices of $V^{\prime}$ and all of the edges in $G$ that contain only vertices from $V^{\prime}$. We use $G-v$ to denote the subgraph induced by $V \backslash\{v\}$.

For an integer $n \geq 1$, we let $K_{n}$ denote the complete graph on $n$ vertices. We will also refer to a complete graph on $n$ vertices as a clique on $n$ vertices. A cycle on $n$ vertices $\left\{v_{1}, v_{2}, \ldots, v_{n}\right\}$ is a graph with edges $E=\left\{\left\{v_{1}, v_{2}\right\},\left\{v_{2}, v_{3}\right\}, \ldots,\left\{v_{n-1}, v_{n}\right\},\left\{v_{n}, v_{1}\right\}\right\}$. Such a cycle will also be referred to as an $n$-cycle or a cycle of length $n$.

Our interest in this work is to consider how positive zero forcing sets are related to cliques in a graph and, further, clique intersection, and clique coverings. Zero forcing on a graph was originally designed to be used as a tool to bound the maximum nullity associated with collections of symmetric matrices derived from a graph $G$ (see, for example, [1]). Positive zero forcing was an adaptation of conventional zero forcing to play a similar role for positive semidefinite matrices (see [3]).

Zero forcing in general is a graph colouring problem in which an initial set of vertices are coloured black, while the remaining vertices are coloured white. Using a designated colour rule, the objective is to change the colour of as many white vertices to black as possible. There are two common rules, these are known as zero forcing and positive zero forcing. The process of a black vertex $u$ changing the colour of a white vertex $v$ to black is usually referred to as " $u$ forces $v$ ". The size of the smallest initial set of black vertices that will "force" all vertices black is called either the zero forcing number or the positive zero forcing number of $G$ depending on which rule is used. This number is denoted by either $Z(G)$ or $Z_{+}(G)$ (again, depending on which rule is used).

Here we are more interested in the behaviour of the positive zero forcing number in connection with cliques and clique coverings in a graph. In particular, we consider $Z_{+}(G)$, when maximal cliques of $G$ satisfy certain intersection properties. Consequently, we now carefully review some basic terminology associated with positive zero forcing in a graph.

The positive zero forcing rule is also based on a colour change rule similar to the zero forcing colour change rule (see [3] and also [5] and [6]). In this case, suppose $G$ is a graph and $B$ a subset of vertices; we initially colour all of the vertices in $B$ black, while all remaining vertices are designated white. Let $W_{1}, \ldots, W_{k}$ be the sets of vertices in each of the connected components of $G$ after removing the vertices in $B$. If $u$ is a vertex in $B$ and $w$ is the only white neighbour of $u$ in the graph induced by the subset of vertices $W_{i} \cup B$, then $u$ can force the colour of $w$ to black. This rule is called the positive colour change rule. The size of the smallest positive zero forcing set of a graph $G$ is denoted by $Z_{+}(G)$. For all graphs $G$, since a zero forcing set is also a positive zero forcing set we have that $Z_{+}(G) \leq Z(G)$. A number of facts have been demonstrated for the positive zero forcing number, see, for example, [3]. If a subset $S$ of $V(G)$ is a positive zero forcing set with $|S|=Z_{+}(G)$, then we refer to $S$ as an optimal positive zero forcing set for $G$.

It is known that by following the sequence of forces throughout the conventional zero forcing process, a path covering of the vertices is derived (see [3, Proposition 2.10] for more details). When the positive colour change rule is applied, two or more vertices can perform 
forces at the same time, and a vertex can force multiple vertices from different components at the same time. This implies that the positive colour change rule produces a partitioning of the vertices into sets of vertex disjoint induced rooted trees, which we will refer to as forcing trees, in the graph.

Given $K_{n}$, the complete graph on $n \geq 2$ vertices, it is not difficult to observe that

$$
Z_{+}\left(K_{n}\right)=Z\left(K_{n}\right)=n-1 .
$$

Furthermore, even though the parameters $Z$ and $Z_{+}$are not generally monotone on induced subgraphs, it is true that if $G$ contains a clique on $k$ vertices, then both $Z(G)$ and $Z_{+}(G)$ are at least $k-1$. So in some sense, cliques in a graph play an important role in determining both zero forcing and positive zero forcing sets in a graph. We explore this correspondence further in this paper. As an example, consider chordal graphs (that is, graph with no induced cycles of length 4 or more). For chordal graphs it is known that the positive zero forcing number is equal to the number of vertices minus the fewest number of cliques that contain all of the edges (see, for example, [7]).

Our paper is divided into twelve sections. The next two sections deal with certain types of clique coverings, followed by two sections devoted to a graph, and its uniqueness, that results from these identified clique coverings, which we call a compressed cliques graph. In the sixth the seventh sections, we discuss the clique covering number of the compressed cliques graph and bounds on the positive zero forcing number. Section 8 connects compressed cliques graphs with certain well-studied Johnson graphs, and Section 9 is concerned with forbidden subgraphs associated with compressed cliques graphs. Following this, the next two sections discuss examples, including a new family of graphs called the vertex-clique graph and a related concept we call the reduced graph, along with additional examples and facts relating the positive zero forcing number and compressed cliques graphs. We conclude with a brief outline for potential future research along these lines.

\section{Simply intersecting clique coverings}

Recall that a clique in a graph is a subset of vertices which induces a complete subgraph. A clique in a graph is maximal if no vertex in the graph can be added to it to produce a larger clique. A clique covering of a graph is a set of cliques with the property that every edge is contained in at least one of the subgraphs induced by one of the cliques in the set. (Note that unless the graph has isolated points, a clique covering that contains every edge also contains every vertex.) The size of a clique covering is the number of cliques in the covering. For a graph $G$, we denote the size of a smallest clique covering by $\operatorname{cc}(G)$. Further, we call a given clique covering minimal if the number of cliques in this covering is equal to $\operatorname{cc}(G)$. Observe that a graph $G$ is a complete graph if and only if $\operatorname{cc}(G)=1$.

A clique covering for a graph $G$ is called a min-max clique covering if its size is $\operatorname{cc}(G)$ and every clique in it is maximal. Let $G$ be a graph and let $\left\{C_{1}, C_{2}, \ldots, C_{\ell}\right\}$ be a min-max clique covering. Any minimal clique covering can be transformed into a min-max clique covering by appropriately adding vertices to the non-maximal cliques.

Proposition 2.1. For any graph $G$, there exists a clique covering with size $\operatorname{cc}(G)$ in which every clique is maximal, that is, there is always a min-max clique covering of $G$. 
We note that there are graphs (e.g. the wheel graph on at least seven vertices) for which there exist multiple clique covers of size $\operatorname{cc}(G)$ in which not all cliques are maximal. Further, a min-max clique covering for a graph may not be unique. Consider the graph, $\operatorname{circ}(6,\{1,2\})$, given in Figure 2. This graph has two min-max clique covers, which are identified in Section 3 .

Let $\mathcal{C}=\left\{C_{1}, \ldots, C_{\ell}\right\}$ be a clique covering for $G$. If for any set of distinct triples $i, j, k \in$ $\{1, \ldots, \ell\}$ it is the case that $C_{i} \cap C_{j} \cap C_{k}=\emptyset$, we say the clique covering has simple intersection. If the clique covering has simple intersection, then a vertex that is in $C_{i} \cap C_{j}$ (where $i \neq j$ ) does not belong to any other clique in $\mathcal{C}$.

There are many examples of graphs with a clique covering with simple intersection. It is not hard to see that the only tree that possesses a clique covering satisfying simple intersection is a path. For a cycle on $n$ vertices, the set of edges forms a min-max clique covering with simple intersection. It turns out that both of the min-max clique covers of $\operatorname{circ}(6,\{1,2\})$ given in the proof of Theorem 3.1 have simple intersection. The wheel on at least seven vertices does not have a minimal clique covering that satisfies simple intersection; the center vertex must belong to at least three cliques in such a clique covering. As a final example, consider the graph in Figure 1. This graph has clique cover number equal to three and a clique cover that satisfies simple intersection, but it has no min-max clique cover that satisfies simple intersection. Throughout this paper, we will only consider the property of simple intersection for min-max clique coverings.

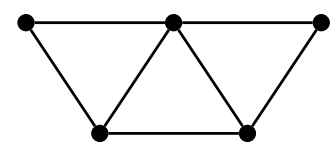

Figure 1: No min-max clique cover of this graph has simple intersection.

\section{Non-unique clique coverings}

Let $G$ be a graph with $n$ vertices, which are labeled $0,1, \ldots, n-1$, and let $S=\left\{k_{1}, k_{2}, \ldots, k_{\ell}\right\}$ be a set of positive integers such that $k_{1}<k_{2}<\cdots<k_{\ell}<(n+1) / 2$. The circulant graph, denoted by $\operatorname{circ}(n, S)$, has each vertex $i$ in $\{0, \ldots, n-1\}$ adjacent to $i \pm k_{1}, i \pm k_{2}, \ldots, i \pm k_{\ell}$ $(\bmod n)$. The graph $\operatorname{circ}(6,\{1,2\})$ plays a key role in identifying graphs that possess a unique min-max clique covering satisfying simple intersection. This graph is isomorphic to the graph in Figure 2. This graph has one vertex for each subset of $\{1,2,3,4\}$ of size two, and two vertices are adjacent if the sets intersect. This graph is also known as the Johnson graph $J(4,2)$ (see also Section 8).

The graph $\operatorname{circ}(6,\{1,2\})$ (see Figure 2 2) has two min-max clique coverings. One clique covering of this graph is $\left\{C_{1}, C_{2}, C_{3}, C_{4}\right\}$ where $C_{i}$ is the set of all vertices with a label that contains an $i$. A second clique covering is formed by the following sets of vertices of 
$\operatorname{circ}(6,\{1,2\})$ :

$$
\begin{array}{ll}
\{\{1,2\},\{1,3\},\{2,3\}\}, & \{\{1,2\},\{1,4\},\{2,4\}\}, \\
\{\{1,3\},\{1,4\},\{3,4\}\}, & \{\{2,3\},\{2,4\},\{3,4\}\} .
\end{array}
$$

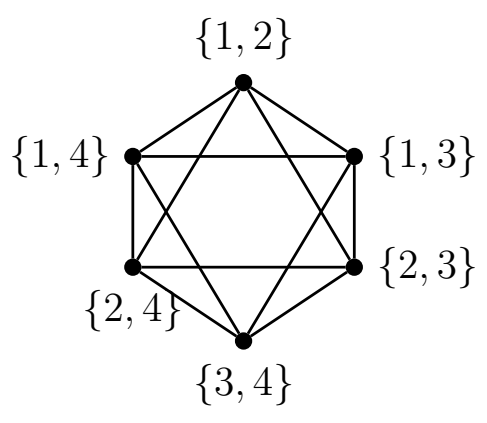

Figure 2: $\operatorname{circ}(6,\{1,2\})$.

The next fact is a key to characterizing the graphs that possess more than one min-max clique covering.

Theorem 3.1. If a graph $G$ has two distinct min-max clique covers that both satisfy simple intersection, then $G$ contains $\operatorname{circ}(6,\{1,2\})$ as an induced subgraph.

Proof. Let $\mathcal{C}=\left\{C_{1}, C_{2}, \ldots, C_{k}\right\}$ be a min-max clique covering for a graph $G$ with simple intersection. Label the vertices in $G$ by the cliques that contain them: if vertex $v$ is in both $C_{i}$ and $C_{j}$, then $v$ gets the label $\{i, j\}$; if $v$ is in $C_{i}$, but no other cliques, then $v$ gets the label $\{i\}$. Distinct vertices may have the same label. Since $\mathcal{C}$ has simple intersection, every vertex has a label with size 1 or 2 . Since every edge must be in at least one clique of $\mathcal{C}$, two vertices are adjacent in $G$ if and only if their labels are intersecting sets. Further, the labels on the vertices in the clique $C_{j}$ must be in the set $\{\{j\},\{j, 1\},\{j, 2\}, \ldots,\{j, k\}\}$.

Assume that $\mathcal{D}=\left\{D_{1}, D_{2}, \ldots, D_{k}\right\}$ is another min-max clique covering of $G$. If there is a vertex in $G$ with the label $\{i\}$, then there is a clique in $\mathcal{D}$ that contains the vertex. Further, every other vertex in this clique must have a label that contains $i$. Since $\mathcal{D}$ is a min-max clique covering, this clique must be maximal and thus must be exactly the clique $C_{i}$, so we know that in this case, $C_{i} \in \mathcal{D}$. Further, if the labels of all the vertices in some $D \in \mathcal{D}$ have a common element, say $i$, then $D=C_{i}$ (again since $D$ is maximal).

Since $\mathcal{C}$ and $\mathcal{D}$ are not equal, we can assume that $C_{1} \notin \mathcal{D}$. This implies that $C_{1}$ does not contain any vertices labeled with $\{1\}$, and $C_{1}$ must contain at least two vertices with distinct labels. Assume that $C_{1}$ contains vertices labeled with $\{1,2\}$ and $\{1,3\}$. The edge between vertices labeled $\{1,2\}$ and $\{1,3\}$ must be in a clique of $\mathcal{D}$, say $D_{a}$. The labels on the vertices in $D_{a}$ must intersect, but not all contain 1 as a common element. Thus we can assume without loss of generality that there are three vertices in $D_{a}$ that have the labels $\{\{1,2\},\{1,3\},\{2,3\}\}$.

We know that the clique $C_{1}$ contains vertices labeled $\{1,2\}$ and $\{1,3\}$. Further, there must be a vertex in $C_{1}$ with the label $\{1, x\}$, where $x \notin\{1,2,3\}$, since if there was no such 
vertex, then any vertex labeled with $\{2,3\}$ could be added to $C_{1}$, this contradicts the fact that $C_{1}$ is maximal (and not in $\mathcal{D}$ ).

Similarly, the clique $C_{2} \in \mathcal{C}$ contains vertices labeled with $\{1,2\}$ and $\{2,3\}$. Since $C_{2}$ must be maximal, it contains another vertex (otherwise we could add the vertex labeled $\{1,3\}$ to it). Assume that this extra vertex is labeled by $\{2, y\}$ (here we could have that $y=2$ ) with $y \neq 1,3$.

Consider the vertex labeled $\{1,2\}$ in the clique covering $\mathcal{D}$. There is an edge between it and the vertex labeled $\{1, x\}$ and the vertex labeled $\{2, y\}$. Since the vertex labeled $\{1,2\}$ is already in the clique $D_{a}$ and $\mathcal{D}$ has simple intersection, these two edges must be in the same clique of $\mathcal{D}$, which implies that $x=y$. Since $x \neq 2,3$, we will assume without loss of generality that $x=y=4$. (Note that $\{1, x\}$ cannot be in the clique $D_{a}$, since $x \notin\{1,2,3\}$ and $\{2, y\} \notin D_{a}$ since $y \neq 1,3$.) Thus there are vertices labeled with $\{1,4\}$ and $\{2,4\}$.

Next consider the clique $C_{3}$, this clique contains vertices labeled by $\{1,3\}$ and $\{2,3\}$. Since $C_{3}$ is maximal and it does not contain the vertex labeled $\{1,2\}$, it must contain a vertex labeled $\{3, z\}$, where $z \neq 1,2$ (but it could be 3 ).

Consider the vertex labeled $\{1,3\}$, this vertex is adjacent to the vertex labeled $\{1,4\}$ and the vertex labeled $\{3, z\}$. In the clique covering $\mathcal{D}$, the vertex labeled $\{1,3\}$ is in $D_{a}$. But neither $\{1,4\}$, nor $\{3, z\}$ are in $D_{a}$ (as $\{1,4\}$ does not intersect with $\{2,3\}$, and $\{3, y\}$ does not intersect with $\{1,2\}$ ). Since $\mathcal{D}$ has simple intersection, $\{1,3\}$ can only be in one other clique. So the vertices labeled with $\{1,4\}$ and $\{3, z\}$ must in this other clique in $\mathcal{D}$. This means that they are adjacent and that $z=4$. Thus there is a vertex labeled by $\{3,4\}$.

It now follows that $G$ contains a subgraph isomorphic to $\operatorname{circ}(6,\{1,2\})$ (see Figure 2).

Unfortunately, the converse to Theorem 3.1 is false in general. A simple example can be derived from the graph $\operatorname{circ}(6,\{1,2\})$ by adding an additional vertex, see Figure 3 , It is easy to verify that this graph has a unique min-max clique covering that satisfies simple intersection, but certainly contains $\operatorname{circ}(6,\{1,2\})$ as an induced subgraph.

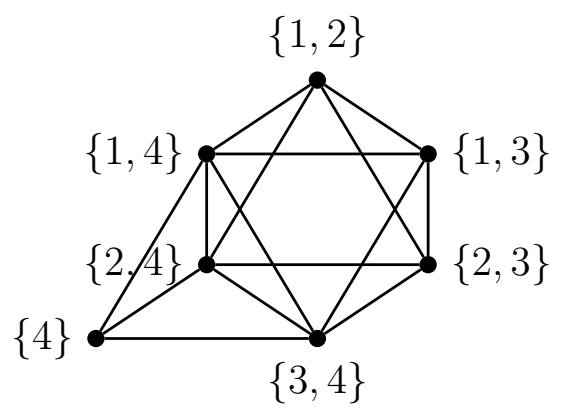

Figure 3: A graph that contains $\operatorname{circ}(6,\{1,2\})$, and has a unique min-max clique cover.

\section{Compressed cliques graphs}

If a graph has a min-max clique covering that satisfies simple intersection, then the graph can be simplified in a way that allows us to determine both the clique covering number and 
the positive zero forcing number of the original graph from the simplified graph.

Let $G$ be a graph and $S=\left\{v_{1}, v_{2}, \ldots, v_{k}\right\}$ a set of vertices in $G$. The contraction of $S$ in $G$ is the graph formed by replacing the vertices in $S$ by a single vertex $v_{S}$, where $v_{S}$ is adjacent to any vertex in $V(G) \backslash S$ that is adjacent to a vertex in $S$.

Let $G$ be a graph with a min-max clique covering $\mathcal{C}=\left\{C_{1}, C_{2}, \ldots, C_{\ell}\right\}$ that has simple intersection. We can construct a new graph that is related to $G$ called a compressed cliques graph. For distinct $i, j \in\{1, \ldots, \ell\}$ define the sets

$$
C_{i, j}=C_{i} \cap C_{j}, \quad C_{i, i}=C_{i} \backslash \bigcup_{\substack{j \in\{1, \ldots, \ell\} \\ j \neq i}} C_{j},
$$

(these sets may be empty). First, for each pair of distinct $i, j \in\{1, \ldots, \ell\}$, if $C_{i, j}$ is nonempty, then contract all the vertices in $C_{i, j}$ to a single vertex labeled $v_{i, j}$. Second, if $C_{i, i}$ is non-empty, then contract all the vertices in $C_{i, i}$ to a single vertex; label this vertex $v_{i, i}$. In this graph the vertices $v_{i, j}$ and $v_{i^{\prime}, j^{\prime}}$ are adjacent if and only if the sets $\{i, j\}$ and $\left\{i^{\prime}, j^{\prime}\right\}$ have non-empty intersection.

We have seen that a graph may have multiple min-max clique covers that satisfy simple intersection. For example the graph $\operatorname{circ}(6,\{1,2\})$. However, for this graph, the compressed cliques graphs, for either clique cover are isomorphic. In fact, both are isomorphic to $\operatorname{circ}(6,\{1,2\})$. Thus, an obvious question is to verify that the associated compressed cliques graphs are isomorphic in the presence of distinct min-max cliques covers satisfying simple intersection (see the next section). Assuming this fact, we denote the compressed cliques graph of $G$ by $\mathcal{C}(G)$ and give some simple examples. For any integer $n$,

$$
\mathcal{C}\left(K_{n}\right)=K_{1}, \quad \mathcal{C}\left(K_{n} \backslash\{e\}\right)=P_{3}(n \geq 3), \quad \mathcal{C}\left(C_{n}\right)=C_{n}, \quad \mathcal{C}\left(P_{n}\right)=P_{n},
$$

(where $K_{n} \backslash\{e\}$ is a complete graph with an edge removed, and $P_{n}$ is the path with $n$ vertices).

Looking at the last two examples, a natural question to ask is when is the compressed cliques graph $\mathcal{C}(G)$ isomorphic to $G$ ?

We will make use of the following map $\phi: V(G) \longrightarrow V(\mathcal{C}(G))$ defined as

$$
\phi(v)= \begin{cases}v_{i, j} & \text { if } v \in C_{i} \cap C_{j}, \\ v_{i, i} & \text { if } v \in C_{i} \text { and no other cliques. }\end{cases}
$$

Theorem 4.1. Let $G$ be a graph with $n$ vertices and $\mathcal{C}=\left\{C_{1}, C_{2}, \ldots, C_{k}\right\}$ be a min-max clique covering of $G$. If $\mathcal{C}$ satisfies simple intersection, then $\mathcal{C}(G)$ isomorphic to $G$ if and only if all of the sets $C_{i, i}$ and $C_{i, j}$ for $i, j \in\{1, \ldots, k\}$ contain no more than one vertex.

Proof. For every $v \in V(G)$, there is a unique pair $(i, j) \in\{1, \ldots, n\} \times\{1, \ldots, n\}$ such that $v \in C_{i, j}$ (it is possible that $i=j$ ).

Consider the map $\phi$ from $V(G)$ to $V(\mathcal{C}(G))$ defined above. If all of the sets $C_{i, i}$ and $C_{i, j}$ for $i, j \in\{1, \ldots, k\}$ contain no more than one vertex this mapping $\phi$ is an edge, and non-edge, preserving bijection. Hence $\mathcal{C}(G) \cong G$.

Conversely, the graph $\mathcal{C}(G)$ has one vertex for each non-empty set $C_{i, j}$. These sets cover the vertices in $G$, so if one of these sets contains two or more vertices, then $\mathcal{C}(G)$ will have less vertices than $G$, and hence these two graphs cannot be isomorphic. 
Corollary 4.2. Suppose $G$ is a graph that possesses a min-max clique covering having simple intersection. Then $\mathcal{C}(\mathcal{C}(G))=\mathcal{C}(G)$.

The map $\phi$ will also be used in Theorem 6.2, there we will need the following fact.

Proposition 4.3. If there is a path from $u$ to $v$ in $G$, then either $\phi(u)=\phi(v)$ or there is a path from $\phi(u)$ to $\phi(v)$ in $\mathcal{C}(G)$.

\section{$5 \quad$ Uniqueness of compressed cliques graphs}

We have seen that the circulant graph $\operatorname{circ}(6,\{1,2\})$ has two distinct min-max clique covers that both satisfy simple intersection - see Figure 2, Furthermore, if we expand each vertex of $\operatorname{circ}(6,\{1,2\})$ to a clique of any positive size and expand edges correspondingly (join the vertices of these cliques if the corresponding vertices were adjacent in $\operatorname{circ}(6,\{1,2\}))$, all of the resulting graphs have two distinct min-max clique covers that both satisfy simple intersection. In order to ensure the concept of a compressed cliques graph well-defined, we need to consider the uniqueness of the compressed cliques graph up to isomorphism. In this section we will show that if a graph has multiple min-max clique covers that satisfy simple intersection, the corresponding compressed cliques graphs are unique, up to isomorphism.

Lemma 5.1. For a graph $G$, let $\mathcal{C}$ be a min-max clique cover of $G$ that satisfies simple intersection. For a vertex $v \in V(G)$ and two distinct cliques $C_{1}, C_{2} \in \mathcal{C}$, we have $v \in C_{1} \cap C_{2}$ if and only if $N_{G}[v]=C_{1} \cup C_{2}$.

Proof. Suppose $v \in C_{1} \cap C_{2}$. Since $\mathcal{C}$ satisfies simple intersection, all neighbours of $v$ must be in $C_{1}$ or $C_{2}$. Thus $N_{G}[v] \subseteq C_{1} \cup C_{2}$. For any vertex $u$ that is in $C_{1}$ or $C_{2}$, since $v \in C_{1} \cap C_{2}$, we know that there is an edge between $v$ and $u$.

On the other hand, suppose $N_{G}[v]=C_{1} \cup C_{2}$. Then $v \in C_{1} \cup C_{2}$. Suppose $v \in C_{1} \backslash C_{2}$. Since $v$ is adjacent to all vertices of $C_{2}$, we know $\{v\} \cup C_{2}$ is also a clique, which contradicts the maximality of $C_{2}$. Hence $v \in C_{1} \cap C_{2}$.

Lemma 5.2. For a graph $G$, let $\mathcal{A}$ and $\mathcal{B}$ be two distinct min-max clique covers of $G$ that both satisfy simple intersection. Then for any vertex $v \in V(G)$, exactly one of the following two possibilities occurs.

1. If only one clique $A$ in $\mathcal{A}$ contains the vertex $v$, then there is a clique $B \in \mathcal{B}$ such that $A=B$.

2. If two distinct cliques $A, A^{\prime} \in \mathcal{A}$ contain the vertex $v$, then there are two distinct cliques $B, B^{\prime} \in \mathcal{B}$ such that $v \in B \cap B^{\prime}$.

Proof. Let $v$ be a vertex of $G$. Since $\mathcal{A}$ is a min-max clique cover of $G$ that satisfies simple intersection, we have the following two cases.

1. The vertex $v$ is contained in exactly one clique $A$ of $\mathcal{A}$. There is a clique $B \in \mathcal{B}$ such that $v \in B$. Suppose that $A \neq B$. Since $A$ and $B$ are maximal cliques, there is a vertex $w \in B \backslash A$. Since $B$ is a clique, $\{v, w\}$ must be an edge in $G$. Since $w \notin A$, the edge $\{v, w\}$ must be covered by a clique in $\mathcal{A} \backslash\{A\}$. Thus, $v$ is in two cliques of $\mathcal{A}$, which is a contradiction. Hence $A=B$. 
2. The vertex $v$ is contained in exactly two distinct cliques $A$ and $A^{\prime}$ of $\mathcal{A}$. If $v$ is contained in exactly one clique $B$ of $\mathcal{B}$, then $A$ is a proper subset of $B$, which contradicts the maximality of $A$. So $v$ is contained in exactly two cliques $B$ and $B^{\prime}$ of $\mathcal{B}$, i.e., $v \in B \cap B^{\prime}$.

Lemma 5.3. For a graph $G$, let $\mathcal{A}$ and $\mathcal{B}$ be two distinct min-max clique covers of $G$ that both satisfy simple intersection. If there are two distinct cliques $A$ and $A^{\prime}$ in $\mathcal{A}$ and two distinct cliques $B$ and $B^{\prime}$ in $\mathcal{B}$ such that $A \cap A^{\prime} \cap B \cap B^{\prime} \neq \emptyset$, then $A \cap A^{\prime}=B \cap B^{\prime}$.

Proof. If one of $A$ or $A^{\prime}$ is equal to one of $B$ or $B^{\prime}$, then it is easy to see that $\left\{A, A^{\prime}\right\}=\left\{B, B^{\prime}\right\}$ and that the result is trivial. Thus we only consider the case that $\left\{A, A^{\prime}\right\} \cap\left\{B, B^{\prime}\right\}=\emptyset$. Suppose $A \cap A^{\prime} \neq B \cap B^{\prime}$. Without loss of generality, let $x \in\left(A \cap A^{\prime}\right) \backslash\left(B \cap B^{\prime}\right)$. Let $v \in A \cap A^{\prime} \cap B \cap B^{\prime}$. It follows from Lemma 5.1 that $A \cup A^{\prime}=N[v]=B \cup B^{\prime}$. So $x$ must be in $B$ or $B^{\prime}$ but cannot be in both.

Since $x \in A \cap A^{\prime}$, from Lemma 5.1 we know that $N_{G}[x]=A \cup A^{\prime}$. Thus $N_{G}[x]=B \cup B^{\prime}$. Furthermore, it follows from Lemma 5.1 that $x \in B \cap B^{\prime}$. This is a contradiction. Therefore $A \cap A^{\prime}=B \cap B^{\prime}$.

Now we can verify that the compressed cliques graph for a graph with a min-max clique covering with simple intersection is well-defined.

Theorem 5.4. If a graph $G$ has a min-max clique cover that satisfies simple intersection, then the compressed cliques graph of $G$ is unique.

Proof. If $G$ has a unique min-max clique cover satisfying simple intersection, it is easy to see that the compressed cliques graph of $G$ is unique. Suppose that $\mathcal{A}=\left\{A_{1}, A_{2}, \ldots, A_{\ell}\right\}$ and $\mathcal{B}=\left\{B_{1}, B_{2}, \ldots, B_{\ell}\right\}$ are two distinct min-max clique covers of $G$ that satisfy simple intersection.

For distinct $i, j \in\{1, \ldots, \ell\}$ define the sets

$$
\begin{aligned}
& A_{i, j}=A_{i} \cap A_{j}, \quad A_{i, i}=A_{i} \backslash \bigcup_{\substack{j \in\{1, \ldots, \ell\} \\
j \neq i}} A_{j}, \\
& B_{i, j}=B_{i} \cap B_{j}, \quad B_{i, i}=B_{i} \backslash \bigcup_{\substack{j \in\{1, \ldots, \ell\} \\
j \neq i}} B_{j} .
\end{aligned}
$$

Let $V_{A}$ be the set of all non-empty sets $A_{i, j}$ with $i, j \in\{1, \ldots, \ell\}$, and $V_{B}$ be the set of all non-empty sets $B_{i, j}$ with $i, j \in\{1, \ldots, \ell\}$. For any $A_{i, i} \in V_{A}$, let $u$ be a vertex in $A_{i, i}$. From the definition, $A_{i, i}$ is the only clique in $\mathcal{A}$ that contains $u$. Thus it follows from Lemma $5.2(1)$ that $A_{i, i} \in V_{B}$. For any $A_{i, j} \in V_{A}(i \neq j)$, let $v$ be a vertex in $A_{i, j}$. From the definition, $A_{i}$ and $A_{j}$ are two distinct cliques in $\mathcal{A}$ that contain $v$. Thus it follows from Lemma $5.2(2)$ that there are two distinct cliques $B_{i^{\prime}}, B_{j^{\prime}} \in \mathcal{B}$ such that $v \in B_{i^{\prime}} \cap B_{j^{\prime}}$. Using Lemma 5.3 we now have $A_{i} \cap A_{j}=B_{i^{\prime}} \cap B_{j^{\prime}}$. Hence $A_{i, j}=B_{i^{\prime}, j^{\prime}} \in V_{B}$. Therefore, $V_{A} \subseteq V_{B}$. Similarly, we can show that $V_{B} \subseteq V_{A}$. So we have $V_{A}=V_{B}$.

Let $E_{A}=\left\{\left\{A_{i, j}, A_{j, k}\right\}: A_{i} \cap A_{j} \neq \emptyset, A_{j} \cap A_{k} \neq \emptyset\right\}$ in which $i, j, k$ are distinct indices, and let $E_{B}=\left\{\left\{B_{i, j}, B_{j, k}\right\}: B_{i} \cap B_{j} \neq \emptyset, B_{j} \cap B_{k} \neq \emptyset\right\}$ in which $i, j, k$ are distinct indices. 
For any $\left\{A_{i, j}, A_{j, k}\right\} \in E_{A}$, there is an edge $\{u, v\} \in E(G)$ such that $u \in A_{i, j}=B_{i^{\prime}, j^{\prime}}$, and $v \in A_{j, k}=B_{j^{\prime \prime}, k^{\prime}}$. Since the edge $\{u, v\}$ is covered by a clique in $\mathcal{B}$, from the simple intersection property for the clique cover $\mathcal{B}$, we know that one of $\left\{B_{i^{\prime}}, B_{j^{\prime}}\right\}$ is the same as one of $\left\{B_{j^{\prime \prime}}, B_{k^{\prime}}\right\}$. Thus $\left\{B_{i^{\prime}, j^{\prime}}, B_{j^{\prime \prime}, k^{\prime}}\right\} \in E_{B}$. So $E_{A} \subseteq E_{B}$. Similarly, we can show that $E_{B} \subseteq E_{A}$, and hence $E_{A}=E_{B}$.

From the definition of the compressed cliques graph, the compressed cliques graph of $G$ with respect to $\mathcal{A}$ is isomorphic to the compressed cliques graph of $G$ with respect to $\mathcal{B}$. Therefore, the compressed cliques graph of $G$ is unique.

Corollary 5.5. Suppose $G$ has two distinct min-max clique covers that both satisfy simple intersection. Then $\mathcal{C}(G)=\operatorname{circ}(6,\{1,2\})$.

Proof. Since $G$ possesses two min-max clique covers that both satisfy simple intersection, we know that $\mathcal{C}(G)$ contains $\operatorname{circ}(6,\{1,2\})$ as an induced subgraph (Theorem 3.1). Assume, for the sake of a contradiction, that $\mathcal{C}(G) \neq \operatorname{circ}(6,\{1,2\})$. Then there exists a vertex $v$ in $\mathcal{C}(G)$ adjacent to some vertex in the subgraph $\operatorname{circ}(6,\{1,2\})$. Referring to Figure 2, suppose $v$ is adjacent to the vertex labeled $\{1,2\}$. (Since $\operatorname{circ}(6,\{1,2\})$ is vertex-transitive, the label $\{1,2\}$ is chosen without loss of generality.) From the hypothesis on $G$ we know that $v$ must also be adjacent to any vertex whose labels contain a 1 or a 2 ; otherwise $G$ does not possess two such clique covers. In this case $\mathcal{C}(G)$ is not a compressed cliques graph as the vertex $v$ should also have the label $\{1,2\}$ and hence did not exist in the first place. By contradiction we conclude that $\mathcal{C}(G)=\operatorname{circ}(6,\{1,2\})$.

Let $\mathcal{G}$ be the set of all graphs that have a min-max clique cover satisfying simple intersection. Let $\mathcal{R}$ be a binary relation on $\mathcal{G}$. We say two graphs $G_{1}, G_{2} \in \mathcal{G}$ have the relation $\mathcal{R}$ if $\mathcal{C}\left(G_{1}\right)=\mathcal{C}\left(G_{2}\right)$. It is easy to see that $\mathcal{R}$ is an equivalence relation, and hence induces a partition of the set $\mathcal{G}$. For any equivalence class that contains a graph $H$, it is easy to see that the graph $\mathcal{C}(H)$ is the minimum element in the class. Thus, the compressed cliques graph of $\mathcal{C}(H)$ is $\mathcal{C}(H)$ itself, see also Corollary 4.2 .

Before we close this section, we note that we could have used the structures derived in the proof of Theorem 5.4 to deduce Corollary 5.5 directly, without relying on Theorem 3.1. Since we chose to consider non-unique min-max clique covers first, we used Theorem 3.1 to establish Corollary 5.5. Observe that if the compressed cliques graph of $G$ is $H$, then $H$ must be an induced subgraph of $G$. Hence if $G$ contains two distinct min-max clique covers that both satisfy simple intersection, we know from Corollary 5.5 that $\mathcal{C}(G)=\operatorname{circ}(6,\{1,2\})$, and hence $G$ contains $\operatorname{circ}(6,\{1,2\})$ as an induced subgraph, which implies Theorem 3.1 .

\section{Clique coverings of compressed cliques graphs}

Before we begin our analysis on positive zero forcing in compressed cliques graphs, we consider clique coverings of compressed cliques graphs. We begin with the following useful lemma. Let $\phi: V(G) \rightarrow V(\mathcal{C}(G))$ be the map defined in (1).

Lemma 6.1. Let $G$ be a graph with a min-max clique covering $\mathcal{C}$ that has simple intersection. If $C$ is a clique in $\mathcal{C}(G)$, then the preimage of $C$ under $\phi$ is a clique in $G$. 
Proof. Assume that $\mathcal{C}=\left\{C_{1}, \ldots, C_{\ell}\right\}$ is a min-max clique covering of $G$ that has simple intersection.

There are two types of cliques in $\mathcal{C}(G)$. The first, are the cliques that are comprised of vertices that correspond to the sets that all contain a common element, say $i$. Clearly the preimage of all such vertices is contained in the clique $C_{i}$.

The second type are the cliques in which the sets do not all contain a fixed element. These cliques must contain exactly three vertices of the form $\{\{a, b\},\{a, c\},\{b, c\}\}$. Let $v$ and $w$ be any two vertices in the preimage of these three vertices. Then $v$ and $w$ must each be in at least two of the cliques $C_{a}, C_{b}, C_{c}$. Thus $v$ and $w$ are both in at least one of these cliques so they are adjacent.

Theorem 6.2. Let $G$ be a graph in which there is a min-max clique covering with simple intersection. Let $\mathcal{C}(G)$ be the compressed cliques graph of $G$. Then

$$
\operatorname{cc}(G)=\operatorname{cc}(\mathcal{C}(G))
$$

Proof. Assume that the clique cover number of $G$ is $\ell$ and that $\left\{C_{1}, C_{2}, \ldots, C_{\ell}\right\}$ is a min-max clique covering with simple intersection.

Let $D_{i}$ be the set of vertices in $\mathcal{C}(G)$ that are labeled $v_{i, j}$ from some $j \in\{1,2, \ldots, \ell\}$ (so $j$ could equal $i$ ). Then $D_{i}$ is a clique in $\mathcal{C}(G)$. We claim that $\left\{D_{1}, D_{2}, \ldots, D_{\ell}\right\}$ is a clique cover for $\mathcal{C}(G)$. The only edges in $\mathcal{C}(G)$ are between vertices $v_{i, j}$ and $v_{i, k}$ for some $i, j, k \in\{1, \ldots, \ell\}$, so the sets $D_{i}$ with $i \in\{1, \ldots, \ell\}$ cover all of the edges of $\mathcal{C}(G)$. Hence the sets $D_{i}$ form a clique cover of $\mathcal{C}(G)$. Thus $\operatorname{cc}(G) \geq \operatorname{cc}(\mathcal{C}(G))$.

On the other hand, let $\mathcal{C}=\left\{C_{1}, C_{2}, \ldots, C_{\ell}\right\}$ be the clique covering of $G$ used to define the compressed cliques graph, and let $\mathcal{D}=\left\{D_{1}, D_{2}, \ldots, D_{\ell}\right\}$ be any minimal clique covering for $\mathcal{C}(G)$. Let $\phi$ be the map defined in (1). We claim that $\left\{\phi^{-1}\left(D_{1}\right), \phi^{-1}\left(D_{2}\right), \ldots, \phi^{-1}\left(D_{\ell}\right)\right\}$ is a clique covering for $G$.

First, if $x, y \in \phi^{-1}\left(D_{a}\right)$, then $\phi(x)$ and $\phi(y)$ are adjacent in $\mathcal{C}(G)$. This implies that $x$ and $y$ are both contained in some clique in $G$. Hence $x$ and $y$ are adjacent in $G$, and $\phi^{-1}\left(D_{a}\right)$ is a clique.

Next, let $e=\{x, y\}$ be any edge in $G$. This edge is covered by a clique in $\mathcal{C}$, say $C_{1}$. Then $\phi(x)=v_{1, a}$ and $\phi(y)=v_{1 . b}$. Thus there is an edge between $\phi(x)$ and $\phi(y)$ in $\mathcal{C}(G)$. This edge is contained in some clique in $\mathcal{D}$, say $D_{a}$. Then both $x$ and $y$ are in $\phi^{-1}\left(D_{a}\right)$, so the edge $\{x, y\}$ is covered. Thus, $\left\{\phi^{-1}\left(D_{1}\right), \phi^{-1}\left(D_{2}\right), \ldots, \phi^{-1}\left(D_{\ell}\right)\right\}$ is a clique covering of $G$ and $\operatorname{cc}(G) \leq \operatorname{cc}(\mathcal{C}(G))$.

We now have the following interesting consequence.

Corollary 6.3. Assume that $G$ is a graph with a min-max clique covering with simple intersection. Let $\left\{D_{1}, \ldots, D_{\ell}\right\}$ be the set of cliques in $\mathcal{C}(G)$ defined such that $D_{i}$ be the set of vertices in $\mathcal{C}(G)$ that are labeled $v_{i, j}$ from some $j \in\{1,2, \ldots, \ell\}$. Then the following statements hold:

1. The set $\left\{D_{1}, D_{2}, \ldots, D_{\ell}\right\}$ forms a min-max clique cover of $\mathcal{C}(G)$; and

2. this clique cover of $\mathcal{C}(G)$ has simple intersection. 
Proof. From Theorem 6.2, the set $\left\{D_{i}\right\}$ forms a minimal clique cover of $\mathcal{C}(G)$. So to prove the first statement, we need to show that each clique $D_{i}$ is maximal. Assume that there is a vertex $u$ in $\mathcal{C}(G)$ that is adjacent to every vertex in $D_{i}$, but is not in $D_{i}$. If $v_{i, i} \in D_{i}$ then, since $u$ is adjacent to $v_{i, i}$, it must be that $u=v_{i, j}$ for some $j$; but in this case the vertex $u$ would be in $D_{i}$. If $D_{i}$ contains the single vertex $v_{i, j}$ (where $i \neq j$ ), then $C_{i} \subseteq C_{j}$, which is a contradiction. Thus we may assume that $D_{i}$ contains the vertices $v_{i, a}, v_{i, b}$. Since $u \notin D_{i}$, this implies that $u=v_{a, b}$, so $D_{i}$ cannot contain any vertices other than these two vertices. In this case, $C_{i}=C_{i, a} \cup C_{i, b}$, and every vertex in $C_{a} \cap C_{b}$ (since $v_{a, b} \in \mathcal{C}(G)$, this intersection is non-empty) is adjacent to every vertex in $C_{i}$. This contradicts $C_{i}$ being a maximal clique.

The second statement follows easily, since if a vertex with label $v_{i, j}$ from $\mathcal{C}(G)$ is contained in three maximal cliques, this contradicts the fact that the original min-max clique covering of $G$ satisfied simple intersection.

\section{$7 \quad$ Positive zero forcing sets in compressed cliques graphs}

Using the strong connections to clique coverings, we now explore positive zero forcing sets and the positive zero forcing number of compressed cliques graphs. This is one of our main motivations for developing this derived graph. Our results in this section are related to the following simple observation about positive zero forcing sets. First note that, by definition, if $N_{G}[u]=N_{G}[v]$, then $u$ and $v$ must be adjacent.

Lemma 7.1. Let $G$ be a graph and $u$ and $v$ be distinct vertices in $G$. If $N_{G}[u]=N_{G}[v]$, then any positive zero forcing set for $G$ will contain at least one of $u$ and $v$.

Proof. Assume that $u$ and $v$ are both initially white in a colouring of $G$. Since $u$ and $v$ are adjacent, they will always be in the same component of $G$ after any set of vertices are removed. Any vertex that is adjacent to one of $u$ or $v$, is adjacent to the other, thus no vertex will ever be able to force either of them.

This can be generalized to a subset of vertices as in the following fact.

Corollary 7.2. Let $G$ be a graph and $\left\{u_{1}, u_{2}, \ldots, u_{k}\right\}$ be a subset of $k$ vertices from $G$. If $N_{G}\left[u_{1}\right]=N_{G}\left[u_{2}\right]=\cdots=N_{G}\left[u_{k}\right]$, then any positive zero forcing set for $G$ will contain at least $k-1$ of the vertices $\left\{u_{1}, u_{2}, \ldots, u_{k}\right\}$.

We can apply the previous result to cliques in a min-max clique covering with simple intersection.

Lemma 7.3. Assume that $G$ is a graph and $\left\{C_{1}, C_{2}, \ldots, C_{\ell}\right\}$ is a min-max clique covering with simple intersection. If $S$ is a positive zero forcing set, then the sets $(V(G) \backslash S) \cap C_{i, j}$ and $(V(G) \backslash S) \cap C_{i, i}$ for any $i, j \in\{1, \ldots, n\}$ have size at most one.

Proof. Assume that there are two vertices $u_{1}, u_{2}$ in $(V(G) \backslash S) \cap C_{i, j}$ (where $i \neq j$ ). Then in the positive zero forcing process in which the vertices in $S$ are initially black, both $u_{1}$ and $u_{2}$ are initially white.

At some point in the positive zero forcing process there is a vertex $v$ which forces $u_{1}$ (assuming that $u_{2}$ is not forced before $u_{1}$ ). Since $u_{1}$ and $u_{2}$ are adjacent, they must be in 
the same component. Since $v$ is adjacent to $u_{1}$, it must be in either $C_{i}$ or $C_{j}$ and thus it is also adjacent to $u_{2}$. But since $u_{2}$ is white at this stage $v$ cannot force $u_{1}$.

A similar argument shows that there cannot be two vertices in $(V(G) \backslash S) \cap C_{i, i}$.

Theorem 7.4. Let $G$ be a graph (that is connected, but not a clique) in which the maximal cliques have simple intersection and let $\mathcal{C}(G)$ be the compressed cliques graph of $G$. Then

$$
|V(G)|-Z_{+}(G)=|V(\mathcal{C}(G))|-Z_{+}(\mathcal{C}(G))
$$

and there exist forcing trees for $G$ and $\mathcal{C}(G)$ that differ only in that these forcing trees for $G$ are isolated vertices.

Proof. Let $S^{\prime}$ be an optimal positive zero forcing set for $\mathcal{C}(G)$; then the vertices in set $V(\mathcal{C}(G)) \backslash S^{\prime}$ are initially coloured white in the positive zero forcing process. Using this set of vertices, we can construct a positive zero forcing set for $G$ as follows. If $v_{i, i}$ is in the set $V(\mathcal{C}(G)) \backslash S^{\prime}$, then colour exactly one of the vertices in $C_{i, i}$ white. Similarly, if $v_{i, j}$ is in the set $V(\mathcal{C}(G)) \backslash S$, then colour exactly one of the vertices in $C_{i, j}$ white. Colour all the vertices that are not white, black. We claim that the set of black vertices is a positive zero forcing set in $G$. To see this, consider that during the positive zero forcing process on $\mathcal{C}(G)$ the vertex $v_{i, j}$ forces the vertex $u_{a, b}$. In this case every vertex in $C_{i, j}$ (in $G$ ) is black and any one of these vertices can force the single white vertex in $C_{a, b}$. This also shows that the set of forcing trees for $G$ and $\mathcal{C}(G)$ only differ in that the set of forcing trees for $G$ may have more isolated vertices.

Thus the number of vertices initially coloured white in $G$ is at least as the number initially colour white in $\mathcal{C}(G)$; that is

$$
|V(G)|-Z_{+}(G) \geq|V(\mathcal{C}(G))|-Z_{+}(\mathcal{C}(G)) .
$$

Conversely, if $S$ is a positive zero forcing set for $G$, then the vertices the $V(G) \backslash S$ are all initially coloured white. Using these vertices it is possible to construct a positive zero forcing set for $\mathcal{C}(G)$. From Lemma 7.3 , there is at most one vertex in $C_{i, j}$ for any pair $i, j \in\{1, \ldots, \ell\}$ that is initially coloured white. If one vertex in $C_{i, j}$ is white, then the vertex $v_{i, j}$ (the representative chosen in the construction of $\left.\mathcal{C}(G)\right)$ in $\mathcal{C}(G)$ is initially coloured white; all other vertices are coloured black.

Assume that at a step in the positive zero forcing process on $G$ the vertex $v$ forces $u$. Then $v$ must be black at this step, and both $v$ and $u$ must both belong to some maximal clique, say $C_{i}$. Consider the following four cases.

Case 1: Assume $u \in C_{i, i}$ and $v \in C_{i, j}$ for some $j$. Since $v$ forces $u$, all the vertices in $C_{i} \backslash u$ must already be black (since they are all adjacent to $v$ ). Any vertex $v_{i, a}$ (except $v_{i, i}$ which is white, since $u$ is white) must be black; since these are the only vertices adjacent to $v_{i, j}$, the vertex $v_{i, j}$ can force $v_{i, i}$ in $\mathcal{C}(G)$.

Case 2: Assume that $u \in C_{i, i}$ and $v \in C_{i, i}$. Then all the vertices in $C_{i}$, except $u$, must be black. When the black vertices are removed, $u$ is an isolated vertex, so it can also be forced by any vertex in $C_{i, j}$. This is Case 1 .

Case 3: Assume $u \in C_{i, j}$ and $v \in C_{i, i}$ for some $j$. Since $v$ can force $u$, all the vertices in $C_{i} \backslash u$ must be black. Thus every vertex $v_{i, a}$, except $v_{i, j}$, must be black in $\mathcal{C}(G)$. The only white neighbour of $v_{i, i}$ is $v_{i, j}$. Hence $v_{i, i}$ can force $v_{i, j}$ in $\mathcal{C}(G)$. 
Case 4: Assume $u \in C_{i, j}$ and $v \in C_{i, k}$ for $j \neq k$. Since $v$ can force $u$, the only white vertex adjacent to $v$ in the same component as $u$ is $u$. This implies that all other vertices in $C_{i, k}$ are black. Thus by Proposition 4.3, $v_{i, j}$ is the only white vertex adjacent to $v_{i, k}$ in its components, so $v_{i, k}$ can force $v_{i, j}$.

Case 5: If $u \in C_{i, j}$ and $v \in C_{i, j}$ for some $j$, then all the vertices in $C_{i}$ and $C_{j}$, except $u$, are black. If the black vertices are removed, then $u$ will be isolated, so any vertex in $\left(C_{i} \cup C_{j}\right) \backslash C_{i, j}$ can force $u$ (this set cannot be empty since $C_{i}$ and $C_{j}$ are distinct, maximal cliques). Then this is either Case 3 or Case 4 .

\section{Connections with Johnson graphs}

The compressed cliques graph is related to the Johnson graph $J(m, 2)$ [4]. The graph $J(m, 2)$ has the set of pairs from $\{1,2, \ldots, m\}$ as its vertex set and two vertices are adjacent if and only if they have non-empty intersection. This graph is the line graph of the complete graph.

We will use a generalization of this graph that we denote by $J^{\prime}(m, 2)$. The vertices of $J^{\prime}(m, 2)$ are the set $\{i, j\}$ and $\{i\}$ where $i, j \in\{1,2, \ldots, m\}$ and two sets are adjacent if and only if they have non-empty intersection. These graphs play a major role in the theory of compressed cliques graphs.

Lemma 8.1. If $G$ is a graph with $\operatorname{cc}(G)>1$ and $G$ has a min-max clique covering that satisfies simple intersection, then the compressed cliques graph of $G$ will be an induced subgraph of $J^{\prime}(\operatorname{cc}(G), 2)$.

There is a simple clique covering for this generalization of the Johnson graph. Define $C_{i}$ to be the set of all vertices in $J^{\prime}(m, 2)$ that contain $i$. Then $C_{i}$ is a maximal clique and $\mathcal{C}=\left\{C_{1}, \ldots, C_{m}\right\}$ is a min-max clique covering that has simple intersection.

Lemma 8.2. For any $m>3$,

1. $\left|V\left(J^{\prime}(m, 2)\right)\right|=\left(\begin{array}{c}m \\ 2\end{array}\right)+m$,

2. $\operatorname{cc}\left(J^{\prime}(m, 2)\right)=m$,

3. $Z_{+}\left(J^{\prime}(m, 2)\right)=\left(\begin{array}{c}m \\ 2\end{array}\right)$,

4. $Z_{+}\left(J^{\prime}(m, 2)\right)=\left|V\left(J^{\prime}(m, 2)\right)\right|-\operatorname{cc}\left(J^{\prime}(m, 2)\right)$.

Proof. The first statement is clear.

For each $i$ define a clique $C_{i}$ that consists of all the vertices that contain $i$; clearly $\left\{C_{1}, C_{2}, \ldots, C_{m}\right\}$ is a clique covering of $J(m, 2)^{\prime}$ of size $m$. Further, each of the edges $\{\{i\},\{i, 1\}\}$ for $i \in\{1,2, \ldots, m\}$ must be in a distinct clique, so the clique cover number cannot be any smaller than $m$.

The set of all vertices of the form $(i, j)$ with $i \neq j$ is a positive zero forcing set-if these vertices are removed, then the remaining graph is an independent set. Thus this positive zero forcing set is optimal from the well known bound $|V(G)|-\operatorname{cc}(G) \leq Z_{+}(G)$ (this is Lemma 11.1). 
For the graph $J^{\prime}(m, 2)$, in an optimal positive zero forcing process there is initially one white vertex for each clique in the min-max clique covering. In fact, we can assign each vertex $\{i\}$ to be white, then within each maximal clique there is exactly one white vertex.

Similarly, the Johnson graph $J(m, 2)$ also has a min-max clique covering with simple intersection (using the same definition for the sets $C_{i}$ ).

Lemma 8.3. For any $m>3$,

1. $V(J(m, 2))=\left(\begin{array}{c}m \\ 2\end{array}\right)$,

2. $\operatorname{cc}(J(m, 2))=m$,

3. $Z_{+}(J(m, 2))=\left(\begin{array}{c}m \\ 2\end{array}\right)-m+2$,

4. $Z_{+}(J(m, 2))=|V(J(m, 2))|-\operatorname{cc}(J(m, 2))+2$.

In this case we need that $m>3$ since the vertices of graph $J(3,2)$ are $\{1,2\},\{1,3\},\{2,3\}$ and these form a complete graph on three vertices. This is an exceptional maximal clique in $J(m, 2)$; every other maximal clique consists of all sets that contain a fixed element. In fact, any maximal clique in the compressed cliques graph with more than three vertices will be the set of all vertices whose corresponding sets all contain a common element.

\section{$9 \quad$ Forbidden subgraphs of compressed cliques graphs}

In the previous section, we observed that the compressed cliques graph is an induced subgraph of the graph $J^{\prime}(m, 2)$, when $m>3$. In this section we illustrate some forbidden subgraphs of the compressed cliques graph.

A claw is a graph with four vertices and three edges in which one vertex is adjacent to the other three (this is the complete bipartite graph $K_{1,3}$ ). The first graph in Figure 4 is a claw. A graph is called claw-free if no set of four vertices induce a subgraph that is a claw. Claw-free graphs have been widely studied, see, for example, the survey [8].

Proposition 9.1. If $G$ is a graph that has a min-max clique covering with simple intersection, then both $G$ and the compressed cliques graph $\mathcal{C}(G)$ are claw-free.

Proof. Let $\mathcal{A}$ be a min-max clique covering of $G$ that satisfies simple intersection. If $G$ has a claw, then the three edges in the claw belong to three different cliques. Thus the center vertex in the claw belongs to three different cliques.

Assume that the compressed cliques graph $\mathcal{C}(G)$ has a claw and that $v_{i, j}$ is the vertex of degree three in the claw. Then each of the other three vertices in the claw must adjacent to $v_{i, j}$, so each will correspond to a set that contains one of $i$ or $j$. This then implies that at least two of the these three vertices will both contain $i$ or will both contain $j$ and thus be adjacent, which is a contradiction.

Note the graph on the right in Figure 4 is special in the sense that it alone cannot be a compressed cliques graph, but rather if a compressed cliques graph contains the subgraph on the right as an induced subgraph, then it must contain other vertices and edges. For example, consider the graph $T_{3}$ given in Figure 5 . 


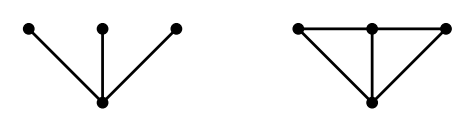

Figure 4: Forbidden induced subgraphs for compressed cliques graphs.

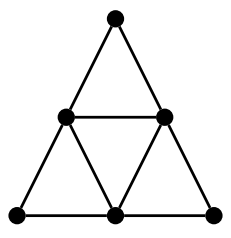

Figure 5: $T_{3}$ : an example of a self-compressed graph.

Observe that the graph $T_{3}$ has clique cover number three, and this min-max clique cover has simple intersection. Furthermore, $\mathcal{C}\left(T_{3}\right)$ is itself $T_{3}$, and includes the graph on the right in Figure 4 as an induced subgraph.

Before we come to our next claim regarding compressed cliques graphs, we define some particular subgraphs. We call a cycle $u_{1} u_{2} \cdots u_{t} u_{1}$ suspended when exactly one of $u_{1}, u_{2}, \ldots, u_{t}$ has degree larger than two in $G$, and all of the remaining vertices from this set have degree two in $G$.

A graph $G$ contains an ear, if it contains $K_{3}$ as a suspended cycle. Observe that if $G$ contains a suspended cycle of length at least four, and it is not just a cycle itself, then $G$ must contain a claw, and hence is forbidden as a compressed cliques graph.

Lemma 9.2. If $G$ is a graph with a min-max clique cover satisfying simple intersection, then $\mathcal{C}(G)$ does not contain a suspended cycle.

Proof. Suppose the compressed cliques graph contains a suspended cycle, from the comments above, the suspended cycle must be an ear. Assume the vertices of the suspended cycle are $u$ and $w$, both of degree two, and $x$ with degree at least three. Then, it follows that at least one of $u$ or $w$ must have a label of distinct indices $\{i, j\}$, namely, $u=v_{i, j}$. In this case, if the label of $w$ is $v_{i, i}$ (or $v_{j, j}$ ), then the label for $x$ must contain an $i$ (since it is adjacent to $w$ ), but also $j$, since other $u$ would be adjacent to another vertex outside of the suspended cycle. This means that both $u$ and $x$ have the same label in $\mathcal{C}(G)$.

If the label of $w$ is given by $w=v_{i, k}$ ( or $v_{j, k}$ ), where $k$ is different from $i$ or $j$, then since $u$ and $w$ have degree two, the label on $x$ must be $\{k, j\}$. Then any other vertex which is adjacent to $x$, must also be adjacent to one of $u$ or $w$.

\section{Vertex-clique graphs}

For any graph $G$ with vertex set $V$ and edge set $E$, we construct a new graph $H$, obtained from $G$, that has a min-max covering with simple intersection. For each vertex $v \in V$, we construct a clique $K_{d(v)}$ where $d(v)$ is the degree of $v$ in $G$. For each edge $\{u, v\} \in E$, we add an edge between a vertex in $K_{d(u)}$ and a vertex in $K_{d(v)}$ such that each vertex in $K_{d(u)}$ (or 
$K_{d(v)}$ ) has at most one neighbour outside of $K_{d(u)}$ (or $K_{d(v)}$ ). This new graph is called the vertex-clique graph of $G$. By definition a vertex-clique graph cannot be a complete graph. The next lemma provides some interesting properties of a vertex-clique graph. Recall that a line graph of a given graph $X$, denoted by $L(X)$, is obtained by associating a vertex with each edge of $X$ and connecting two vertices with an edge if and only if the corresponding edges of $X$ have a vertex in common.

Lemma 10.1. Let $H$ be a vertex-clique graph of $G$. Then

1. $H$ is a line graph;

2. H has a min-max clique covering that satisfies simple intersection; and

3. $\mathcal{C}(H)=H$.

Proof. If $H$ is the vertex-clique graph of $G$, then $H$ is the line graph of the graph formed by performing an edge subdivision on each edge of $G$.

Let $C_{v}$ be the clique in $H$ that replaces the vertex $v$ in $G$, similarly, let $C_{\{u, v\}}$ be the edge in $H$ between a vertex in $C_{v}$ and $C_{u}$. The set of all $C_{v}$ and $C_{\{u, v\}}$ form a min-max clique covering that satisfies simple intersection.

Finally we show that $\mathcal{C}(H)=H$. Note that the set $C_{v} \cap C_{u}$ is empty, and $C_{v} \cap C_{\{u, v\}}=\{v\}$. Consider the set $C_{\{u, v\}} \cap C_{\left\{u^{\prime}, v^{\prime}\right\}}$. If $C_{\{u, v\}}$ is an edge in $H$, then there is no edge $C_{\left\{u^{\prime}, v\right\}}$, so any $C_{\{u, v\}}$ and $C_{\left\{u^{\prime}, v^{\prime}\right\}}$ will be disjoint. Thus the intersection of any two cliques in this covering is either empty, or has size one. By Theorem 4.1, this implies that $\mathcal{C}(H)$ is isomorphic to $H$.

For a graph $G$, we can form a simplified graph called the reduced graph of $G$ that has the same positive zero forcing number as the original graph. First we define an induced path $u_{1} u_{2} \cdots u_{t}$ in a graph to be a suspended path if the vertices $u_{2}, u_{3}, \ldots, u_{t-1}$ all have degree two. To form the reduced graph of $G$, first recursively delete all vertices with degree one. Once all the vertices of degree one have been removed, contract any induced suspended paths of length at least two to an edge. The graph that remains after performing both of these operations is called the reduced graph of $G$, and is denoted by $\mathcal{R}(G)$. It is clear that these two operations do not effect the size of a positive zero forcing set.

Lemma 10.2. Let $G$ be a graph, then $Z_{+}(G)=Z_{+}(\mathcal{R}(G))$.

Applying this reduction to a vertex-clique graph produces a new graph for which we can determine bounds on the positive zero forcing number.

Theorem 10.3. Let $G$ be a connected vertex-clique graph and $\mathcal{R}(G)$ be the reduced graph of G.

1. If $\mathcal{R}(G)$ has only one vertex, then $Z_{+}(G)=1$.

2. If $\mathcal{R}(G)$ is a 3-cycle, then $Z_{+}(G)=2$.

3. If $\mathcal{R}(G)$ has more than three vertices, let $k$ be the number of edges in $\mathcal{R}(G)$ that are themselves maximal cliques in $\mathcal{R}(G)$, then $Z_{+}(G) \leq k$. Further, this upper bound can be tight for some graphs $G$. 
Proof. Note that each cycle in $G$ is either unchanged in $\mathcal{R}(G)$ or reduced to a cycle of smaller length. If $\mathcal{R}(G)$ has only one vertex, then $G$ is a path, and $Z_{+}(G)=1$. If $\mathcal{R}(G)$ is a 3-cycle, then $G$ is a unicyclic graph, and $Z_{+}(G)=2$.

Suppose that $\mathcal{R}(G)$ has more than three vertices. Let $C^{(2)}$ be the set of all maximal cliques of size two in $\mathcal{R}(G)$, and let $C^{(\geq 3)}$ be the set of all maximal cliques of size at least three in $\mathcal{R}(G)$. Since $G$ is a connected vertex-clique graph, we know that every vertex in $\mathcal{R}(G)$ must belong to exactly two maximal cliques, one in $C^{(2)}$ and the other in $C^{(\geq 3)}$. Note that $C^{(\geq 3)} \neq \emptyset$ as $\mathcal{R}(G)$ has more than three vertices. Color all vertices of $\mathcal{R}(G)$ using the following procedure.

1. Pick a clique $C \in C^{(\geq 3)}$, color all vertices of $C$ black. For each edge in $C^{(2)}$, if one endpoint is black and the other is not coloured, then color the uncoloured vertex to grey.

2. If there is a clique $C \in C^{(\geq 3)}$ that contains both grey and uncoloured vertices, then color all uncoloured vertices of $C$ to black.

3. For each edge in $C^{(2)}$, if one endpoint is black and the other is uncoloured, then color the uncoloured vertex to grey.

4. If all vertices of $\mathcal{R}(G)$ are coloured, then stop; otherwise, go to Step 2 .

In the above procedure, when a vertex $u$ is coloured black, there must be a neighbour $v$ such that $\{u, v\} \in C^{(2)}$ and $v$ is coloured grey because every vertex in $\mathcal{R}(G)$ belongs to exactly two maximal cliques (one in $C^{(2)}$ and the other in $C^{(\geq 3)}$ ). Thus, every black vertex in $\mathcal{R}(G)$ is an endpoint of an edge in $C^{(2)}$. On the other hand, for each edge in $C^{(2)}$, at most one endpoint is coloured black. As all black vertices form a positive zero forcing set of $\mathcal{R}(G)$, it follows from Lemma 10.2 that $Z_{+}(G)=Z_{+}(\mathcal{R}(G)) \leq\left|C^{(2)}\right|$.

Finally, we give an example that shows the upper bound in the theorem above cannot be improved in general. Let $H$ be the vertex-clique graph of complete bipartite graph $K_{2,3}$ (see Figure 6). Then $\mathcal{R}(H)=K_{2} \square K_{3}$, where $\square$ denotes the Cartesian product of graphs. Note that the number of edges in $K_{2} \square K_{3}$ that are themselves maximal cliques is three, and $Z_{+}(H)=3$. In general, if $H$ is the vertex clique graph of $K_{2, n}$, then $\mathcal{R}(H)=K_{2} \square K_{n}$. In this case, $Z_{+}(\mathcal{R}(H))=n$ and the number of edges in $\mathcal{R}(H)$ that form maximal cliques is precisely $n$.
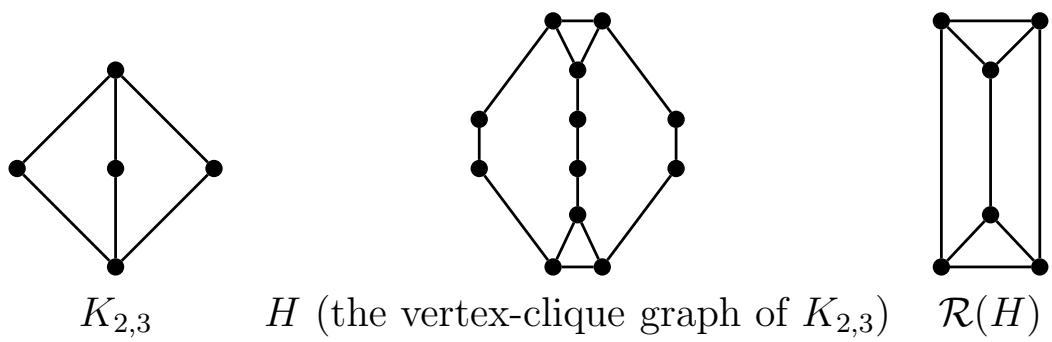

Figure 6: Example of a graph where the bound in Theorem 10.3 is tight. 


\section{Examples of compressed cliques graphs}

We now consider several examples of compressed cliques graphs $G$ with an eye on comparing the values of $Z_{+}(G)$ and $|V(G)|-\operatorname{cc}(G)$. Our focus on these graphs comes from the following well known lemma (see also [3]).

Lemma 11.1. For any graph $G$

$$
|V(G)|-\operatorname{cc}(G) \leq Z_{+}(G)
$$

Our first result gives a large family for graphs for which the positive zero forcing number is equal to the difference between the number of vertices and the clique covering number. If $\mathcal{C}(G)$ contains no induced cycles (other than $K_{3}$ ), then $G$ is a chordal graph in which a min-max clique covering satisfies simple intersection. Theorem 4.6 .5 of [2] states that for a chordal graph $G, Z_{+}(G)=|V(G)|-\operatorname{cc}(G)$. Thus we have the following result.

Theorem 11.2. If $G$ has a min-max clique cover with simple intersection and the compressed cliques graph has no induced cycles, other than $K_{3}$, then

$$
Z_{+}(G)=|V(G)|-\operatorname{cc}(G)
$$

If $G$ consists of a series of cliques $C_{1}, \ldots, C_{k}$ in which only consecutive cliques intersect, then we call $G$ a path of cliques. In this case we have equality in the inequality of Lemma 11.1.

Corollary 11.3. Let $G$ be a graph that is a path of cliques, then

$$
|V(G)|-\operatorname{cc}(G)=Z_{+}(G)
$$

The next family of graphs that we consider are a generalization of the musical graph $M_{n}$ defined in [9]. For $n \geq 3$, the graph $M_{n}$ has $2 n$ vertices, $5 n$ edges, and is isomorphic to the Cayley graph $\operatorname{Cay}\left(\mathbb{Z}_{2 n},\{ \pm 1, \pm(n-1), n\}\right)$. Figure [7 is the musical graph $M_{4}$.

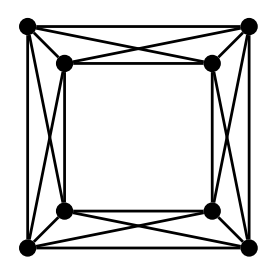

Figure 7: The musical graph $M_{4}$.

The graph $M_{n}$ has a min-max clique covering with $n$ cliques that satisfies simple intersection. The positive zero forcing number for $M_{n}$ can then be easily calculated.

Lemma 11.4. Let $n \geq 3$, then $Z_{+}\left(M_{n}\right)=n+2$.

Proof. Observe that the compressed cliques graph of musical graph $M_{n}$ is $C_{n}$. Therefore, using Theorem 7.4 we have

$$
\left|V\left(M_{n}\right)\right|-Z_{+}\left(M_{n}\right)=\left|V\left(\mathcal{C}\left(M_{n}\right)\right)\right|-Z_{+}\left(\mathcal{C}\left(M_{n}\right)\right)=n-2
$$

and so $Z_{+}\left(M_{n}\right)=n+2$. 
The musical graph is an example of a graph for which

$$
Z_{+}(G) \leq|V(G)|-|\operatorname{cc}(G)|+2 .
$$

We can generalize this to a family of related graphs. Let $G$ be a graph for which $\left\{C_{1}, C_{2}, \ldots, C_{\ell}\right\}$ is a min-max clique covering. If each of $C_{i} \cap C_{i+1}$ for $i=1, \ldots, \ell-1$, as well as $C_{1} \cap C_{\ell}$, are non-empty (these are the consecutive cliques), while all other intersections of these cliques are empty, then $G$ is called a cycle of cliques. These graphs are a generalization of the graphs defined as $C_{t}\left(K_{n}\right)$ [10].

Theorem 11.5. Let $G$ be a cycle of cliques, then

$$
Z_{+}(G) \leq|V(G)|-\operatorname{cc}(G)+2 .
$$

Proof. Assume that $G$ is a cycle of cliques $\left\{C_{1}, C_{2}, \ldots, C_{\ell}\right\}$. For $i=2,3, \ldots, \ell-1$, let $v_{i}$ be a vertex in $C_{i} \cap C_{i+1}$ (since $G$ is a cycle of cliques, this is possible). Colour the vertices $v_{2}, \ldots, v_{\ell-1}$ white and all other vertices in $G$ black. We claim that this is a positive zero forcing set for $G$. To see this, note that any vertex in $C_{1} \cap C_{2}$ can force $v_{2,3}$, which in turn forces $v_{3,4}$. Continuing in this manner, all the vertices in $G$ will be forced to black.

There is a subfamily of the cycles of cliques for which the positive zero forcing number is equal to the number of vertices minus the clique covering number of the graph.

Theorem 11.6. Let $G$ be a cycle of cliques labeled $\left\{C_{1}, C_{2}, \ldots, C_{\ell}\right\}$. If there are two cliques $C_{i}$ and $C_{j}$ with $C_{i, i}$ and $C_{j, j}$ non-empty, then

$$
Z_{+}(G)=|V(G)|-\operatorname{cc}(G) .
$$

Proof. Assume $i$ is the smallest $i$ such that $C_{i, i}$ is non-empty and $j$ is the largest value for which $C_{j, j}$ is non-empty.

The clique compressed cliques graph for $G$ will contain a cycle with vertices labeled $v_{i, i+1}$ for $i=1, \ldots, \ell-1$ and $v_{1, \ell}$. Since the sets $C_{i, i}$ and $C_{j, j}$ are non-empty the graph will also have vertices $v_{i, i}$ (adjacent to $v_{i-1, i}$ and $v_{i, i+1}$ ), and $v_{j, j}$ (adjacent to $v_{j-1, j}$ and ${ }_{j, j+1}$ ).

Colour the vertices $v_{i, i}$ and $v_{j, j}$ white along with every vertex $v_{k, k+1}$ in the cycle, except the vertices in the clique $C_{j}$. The remaining vertices are coloured black. In this colouring, there are $\ell$ white vertices, so the number of black vertices is $|V(G)|-\ell$. We show that the black vertices form a positive zero forcing set for $G$.

The vertex $v_{j, j+1}$ will force $v_{j, j}$ and then start a forcing sequence by forcing $v_{j+1, j+2}$ and continuing to $v_{i-1, i}$. Similarly, $v_{j-1, j}$ will force the vertex $v_{j-2, j-1}$, and continue forcing along the cycle until the vertex $v_{i, i+1}$. Finally, $v_{i, i+1}$ can force $v_{i, i}$.

Our next example is a family of graphs for which there is a large gap between the positive zero forcing number and the number of vertices minus the clique covering number of the graph.

Define a graph $X\left(n ; \ell_{1}, \ldots, \ell_{k}\right)$ that has vertices $x_{1}, \ldots, x_{n}$ which induce a clique. In addition, this graph also contains disjoint cycles $C_{1}, \ldots, C_{k}$, in which $C_{i}$ has length $\ell_{i}$, and each cycle contains exactly two vertices from the set $\left\{x_{1} \ldots, x_{n}\right\}$ (see also Figure 8 where $X(8 ; 4,4,4,4)$ is depicted). In this case, the number of vertices in this graph is $n+\sum_{i=1}^{k}\left(\ell_{i}-2\right)$ and $\operatorname{cc}(X)=1+\sum_{i=1}^{k}\left(\ell_{i}-1\right)$. Moreover, the only such clique cover is a min-max clique cover that has simple intersection. 


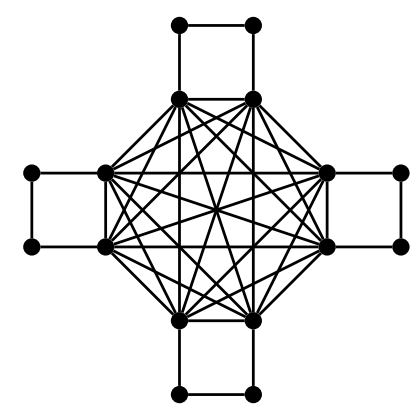

Figure 8: The graph $X(8 ; 4,4,4,4)$.

Theorem 11.7. Let $n, \ell_{1}, \ldots, \ell_{k}$ be integers with $n \geq \sum_{i} \ell_{i}$. Then $X=X\left(n ; \ell_{1}, \ldots, \ell_{k}\right)$ has a min-max clique covering with simple intersection such that

$$
Z_{+}\left(X\left(n ; \ell_{1}, \ldots, \ell_{k}\right)\right)=n-1=|V(X)|-\operatorname{cc}(X)+k .
$$

Proof. To verify this, colour all but one of the vertices in the $n$-clique black.

\section{Further work}

We have shown that for graphs that have a clique covering satisfying simple intersection, we can determine the positive zero forcing number of the graph from the positive zero forcing number of the compressed cliques graph. Further, any compressed cliques graph will be an induced subgraph of the generalization of the Johnson graph $J^{\prime}(n, 2)$ given in Section 8 . This graph plays a special role in this theory.

In this paper, we defined a simple intersecting clique covering of a graph. This means that any vertex of the graph is contained in at most two cliques in the covering. Clearly, this can be generalized. We will say a clique covering of a graph has s-wise intersection if any vertex is contains in at most $s$ cliques in the clique covering. Then we could generalize the Johnson graph $J(n, s)$, to the graph $J^{\prime}(n, s)$. The vertices of this graph will be subsets of size at most $s$ from $\{1, \ldots, n\}$ and two vertices will be adjacent if and only if there sets are intersecting. Then every graph will have a clique covering that has $s$-wise intersection for $s$ sufficiently large. Then for any graph $G$, the compressed cliques graph of $G$ is an induced subgraph of $J^{\prime}(n, s)$. Moreover, if we can determine the positive zero forcing number for the compressed cliques graph, then we can determine the positive zero forcing number for the original graph $G$.

\section{References}

[1] AIM Minimum Rank-Special Graphs Work Group. Zero forcing sets and the minimum rank of graphs. Linear Algebra Appl., 428(7): 1628-1648, 2008.

[2] Fatemeh Alinaghipour Taklimi. Zero Forcing Sets for Graphs. Ph.D. Thesis, University of Regina, 2013. 
[3] F. Barioli, W. Barrett, S. Fallat, H.T. Hall, L. Hogben, B. Shader, P. van den Driessche, and $\mathrm{H}$. van der Holst. Zero forcing parameters and minimum rank problems. Linear Algebra Appl., 433(2): 401-411, 2010.

[4] M. Doob. Spectral Graph Theory. In Handbook of Graph Theory. J.L. Gross and J. Yellen, Editors. CRC Press, Boca Raton, FL, 2004.

[5] J. Ekstrand, C. Erickson, H.T. Hall, D. Hay, L. Hogben, R. Johnson, N. Kingsley, S. Osborne, T. Peters, J. Roat, et al. Positive semidefinite zero forcing. Linear Algebra Appl., 439: 1862-1874, 2013.

[6] J. Ekstrand, C. Erickson, D. Hay, L. Hogben, and J. Roat. Note on positive semidefinite maximum nullity and positive semidefinite zero forcing number of partial 2-trees. Electron. J. Linear Algebra, 23: 79-97, 2012.

[7] S. Fallat, K. Meagher, and B. Yang. On the Complexity of the Positive Semidefinite Zero Forcing Number. Accepted in Linear Algebra Appl., (2015) http://dx.doi.org/10.1016/j.laa.2015.03.011.

[8] R. Faudree, E. Flandrin, and Z. Ryjacek. Claw-free graphs - A survey. Discrete Math., 164: 87-147, 1997.

[9] D.E. Knuth. The Art of Computer Programming. Vol.4, Introduction to Combinatorial Algorithms and Boolean Functions, Addison-Wesley Professional, New York, 2008.

[10] T.A. Peters. Positive semidefinite maximum nullity and zero forcing number. Ph.D. Thesis, Iowa State University, 2012. 